\title{
Identification of putative drugs against viral respiratory infections by the pharmacovigilance analysis tool OpenVigil 2
}

\author{
Ruwen Böhm ${ }^{1}$, Claudia Bulin ${ }^{1}$, Vicki Wätzig ${ }^{1}$, Ingolf Cascorbi ${ }^{2}$, Hans-Joachim Klein ${ }^{3}$, and \\ Thomas Herdegen ${ }^{1}$ \\ ${ }^{1}$ University Medicine Schleswig-Holstein \\ ${ }^{2}$ University Hospital Schleswig-Holstein \\ ${ }^{3}$ Kiel University
}

June 16, 2020

\begin{abstract}
Aim: Pharmacovigilance data are primarily used to identify adverse drug reactions. However, scanning for associations of drugs and adverse events that occur less frequently than expected provides hypotheses for drug repurposing, i.e. a known drug could be therapeutically beneficial for a new indication like the coronavirus disease (COVID-19). Methods: Drugs associated with viral respiratory tract infections and/or influenza were extracted from the U.S. FAERS pharmacovigilance data using OpenVigil2.1MedDRA17, filtered for significant inverse associations (punadj<0.05), checked for plausibility, and categorised by their WHO Anatomical Therapeutic Chemical (ATC) classification code. Results: ATC clustering of 82 candidate drugs revealed antidiabetics, neuropharmacologic sigma-receptor agonists, peptidase inhibitors, kinase inhibitors and anti-androgens. Chloroquine appears as a statistically significant risk factor for viral diseases supporting actual knowledge. Conclusion: OpenVigil 2 delivers new hypotheses for drug repurposing, theoretically for all indications. There is affirmative data for some of our results; the remaining proposed candidate drugs without already known antiviral mechanism of action should stimulate further exploration.
\end{abstract}

\section{Hosted file}

manuscript.pdf available at https://authorea.com/users/333943/articles/460037-identificationof-putative-drugs-against-viral-respiratory-infections-by-the-pharmacovigilanceanalysis-tool-openvigil-2 


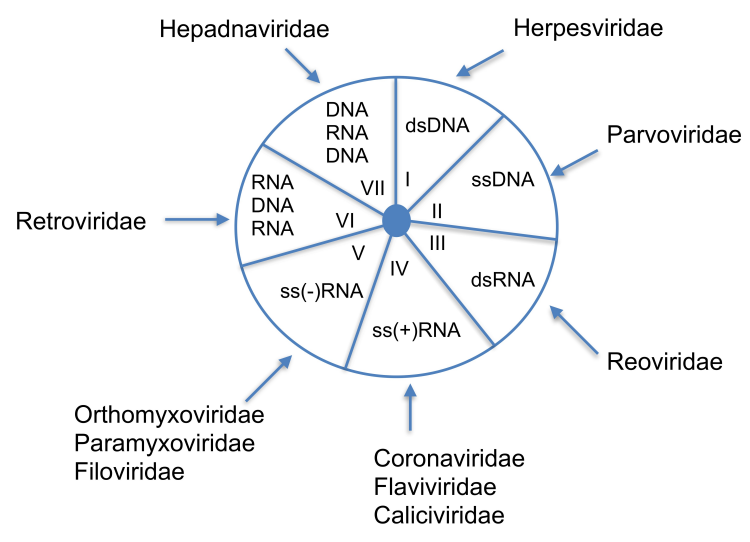

A

B

\begin{tabular}{|c|c|c|c|c|c|c|c|}
\hline & + + Drug & - drug & |Sums & & Drug $(\mathbf{s})$ of interest & All other druos & \\
\hline + Adverse Event & (DE) & (dE) & $\mathrm{E}$ & Adverse event(s) of interest & 6 & 614 & 620 \\
\hline - adverse event & (D) & (de) & e & All other adverse events & 25986 & 6692804 & 6718790 \\
\hline Sums & $\mathrm{D}$ & d & $\mathrm{N}$ & $\Sigma$ & 25992 & 6693418 & 6719410 \\
\hline
\end{tabular}

$\backslash$

Rate (DE/D): $0.023084 \%$

Chi-Squared with Yates' correction: 4.027426

Interpretation: Do the observed frequencies differ from expected frequencies? The greater the chi-squared value, the greater the differences. Chi square values greater than 4 are considered statistically significant.

Measurements of disproportionality (observed-expected ratios like RRR, PRR, ROR)

Interpretation: Generally, the higher the value, the more likely an association between drug(s) and adverse event(s) has been found intervals can be used instead of the chi-squared value above to assecure statistical significance.

Relative Reporting Ratio (RRR) and 95\% confidence interval (lower bound; upper bound): 2.501791 ( $1.11971 ; 5.589801$

Proportional Reporting Ratio (PRR) and 95\% confidence interval (lower bound; upper bound): 2.516466 ( $1.126236 ; 5.622802$ )

Reporting Odds Ratio (ROR) and 95\% confidence interval (lower bound; upper bound): 2.516816 ( $1.126185 ; 5.624623$ )

According to the criteria of Evans 2001 ( $\mathrm{n}>3$, chisq $>4$, PRR $>2$ ) this combination of drug $(\mathrm{s})$ and adverse event( $\mathrm{s})$ is considered: likely an adverse reaction 

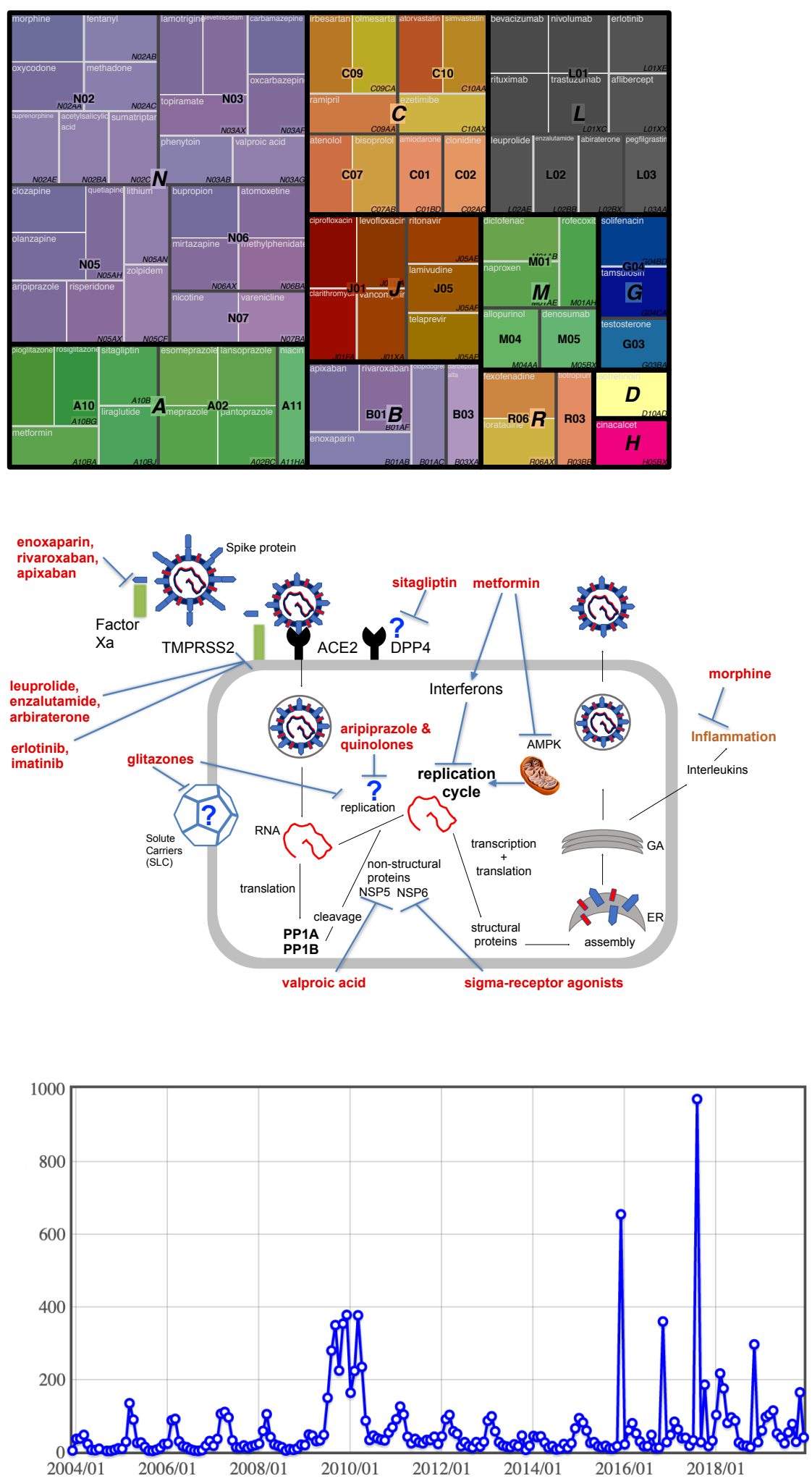